\title{
Validação de Modelos ER
}

\author{
Cody Malnor ${ }^{1,2}$,André Chateaubriand ${ }^{1}$, Obede Carvalho ${ }^{1}$, Ricardo Terra ${ }^{1}$ \\ ${ }^{1}$ Departamento de Ciência da Computação \\ Universidade Federal de Lavras (UFLA) \\ ${ }^{2}$ Department of Mathematics and Computer Science \\ Northern Michigan University (NMU) \\ cmalnor@nmu.edu, \{andrechateau, obedejc\}@computacao.ufla.br, \\ terra@dcc.ufla.br
}

\begin{abstract}
The Entity-Relationship (ER) model is widely used for teaching conceptual data modeling. During the learning process, many of the mistakes made by students are not related to the logic of the model, but to its construction. In this paper, we propose and formalize an ER model validation integrated to an academic modeling tool. This extension checks connections as soon as they are added to the model and - if invalid - not only notifies the student but also suggests the correct connections. As a contribution, we seek to make the learning process faster and more interactive for the student, in addition to reducing the teacher's correction effort.
\end{abstract}

Resumo. O modelo Entidade-Relacionamento (ER) é largamente adotado no ensino de modelagem de dados conceitual. Durante o processo de aprendizagem, muitos dos erros cometidos pelos alunos não estão relacionados à lógica do modelo, mas sim às suas construções. Diante disso, este artigo propõe e formaliza uma validação de modelos ER integrada à uma ferramenta acadêmica de modelagem. Esse módulo de validação é capaz de verificar conexões logo que são adicionadas ao modelo e - caso inválidas - não só notificar o aluno como sugerir as conexões corretas. Como contribuição, busca-se tornar o processo de aprendizado mais rápido e interativo para o aluno, além de reduzir o esforço de correção pelo professor.

\section{Introdução}

O modelo Entidade-Relacionamento (ER) é uma das abordagens de modelagem de dados conceitual mais adotadas no ensino de disciplinas de banco de dados [2, 16]. Esse modelo é baseado em uma percepção de um mundo real construída por uma coleção de objetos básicos, chamados entidades, e os relacionamentos entre esses objetos. Existem uma ampla gama de ferramentas que auxiliam a criação de modelos ER, tais como Dia ${ }^{1}$, TerraER ${ }^{2}$ EERCASE ${ }^{3}$ e MySQLWorkbench ${ }^{4}$

No modelo ER, obviamente, não é permitido qualquer tipo de conexão entre quaisquer dois de seus elementos. Isso implica em alunos - durante o processo de aprendizagem - cometendo uma série de erros não relacionados à lógica do modelo, mas sim, às

\footnotetext{
${ }^{1}$ http://www.dia-installer.de

${ }^{2} \mathrm{http}: / /$ www.terraer.com.br

${ }^{3}$ https://www.sites.google.com/a/cin.ufpe.br/eercase/apresentacao

${ }^{4}$ https://www.mysql.com/products/workbench/
} 
suas construções. Mais importante, esses erros podem demorar para serem identificados como também corrigidos. Este artigo, portanto, reivindica que alunos devem focar na tarefa de modelagem conceitual, sem preocupações com formalizações do modelo.

Diante disso, este artigo propõe uma validação de modelos ER integrada à uma ferramenta acadêmica de modelagem, denominada TerraER. Esse módulo de validação é capaz de verificar conexões logo que são adicionadas ao modelo e - caso inválidas - não só notificar o aluno como sugerir as conexões válidas por meio de uma tela de recomendação que aponta quais as possíveis conexões entre os elementos e ainda quais os possíveis elementos que podem ter tal conexão estabelecida.

Como contribuição, a validação proposta neste artigo visa tornar o processo de aprendizado mais rápido e interativo para o aluno, além de reduzir o esforço de correção pelo professor. Isso se justifica, primeiramente, pelo fato de o módulo de validação permitir o erro do aluno e imediatamente o notificar; cenário o qual instiga o aprendizado e evita a recorrência do mesmo erro. Em segundo lugar, o aluno pode se preocupar exclusivamente na tarefa de modelagem conceitual, sem preocupações com formalizações do modelo. Da mesma forma, professores, no processo de correção, podem ter a atenção voltada exclusivamente à modelagem, o que fomenta maior eficiência do processo educacional.

O artigo está dividido como a seguir. A Seçãon 2 introduz os conceitos fundamentais à compreensão deste estudo. A Seção 3 formaliza as conexões válidas do modelo ER, descreve o projeto e implementação da validação integrada à ferramenta TerraER e conduz uma avaliação sob a perspectiva educacional. Por fim, a Seção 4 conclui o estudo.

\section{Background}

Esta seção apresenta conceitos fundamentais ao entendimento deste estudo, tais como modelo ER, modelo ER estendido (EER) e a ferramenta de modelagem conceitual de alto nível TerraER.

\subsection{Modelo ER}

Segundo Garcia-Molina [7], no modelo Entidade-Relacionamento, o esquema do banco de dados é representado graficamente como um diagrama usando três principais tipos de elementos: entidades, atributos e relacionamentos.

Entidade: Uma entidade é um objeto abstrato de algum tipo, e uma coleção de entidades similares forma um conjunto de entidades [7]. Chen [2] classifica essas entidades como entidades fortes e entidades fracas. Uma entidade fraca é uma entidade cuja existência depende de alguma forma de outra entidade, de tal forma que sua existência não se justifica sem que essa outra entidade (denominada entidade proprietária) exista. Como exemplo, a Figura 1 apresenta a entidade DEPENDENTE na qual depende sua existência da entidade FUNCIONARIO, pois um dependente demanda vínculo com o funcionário e não se justifica sem ele. Ao contrário, uma entidade forte $\mathrm{f}^{5}$ é uma entidade que não é fraca, ou seja, a entidade FUNCIONARIO da Figura 1 não depende de nenhuma outra para existir.

\footnotetext{
${ }^{5}$ Alguns autores utilizam o termo "entidade regular" ao invés de "entidade forte" [3], porém neste trabalho é adotado o termo "entidade forte".
} 


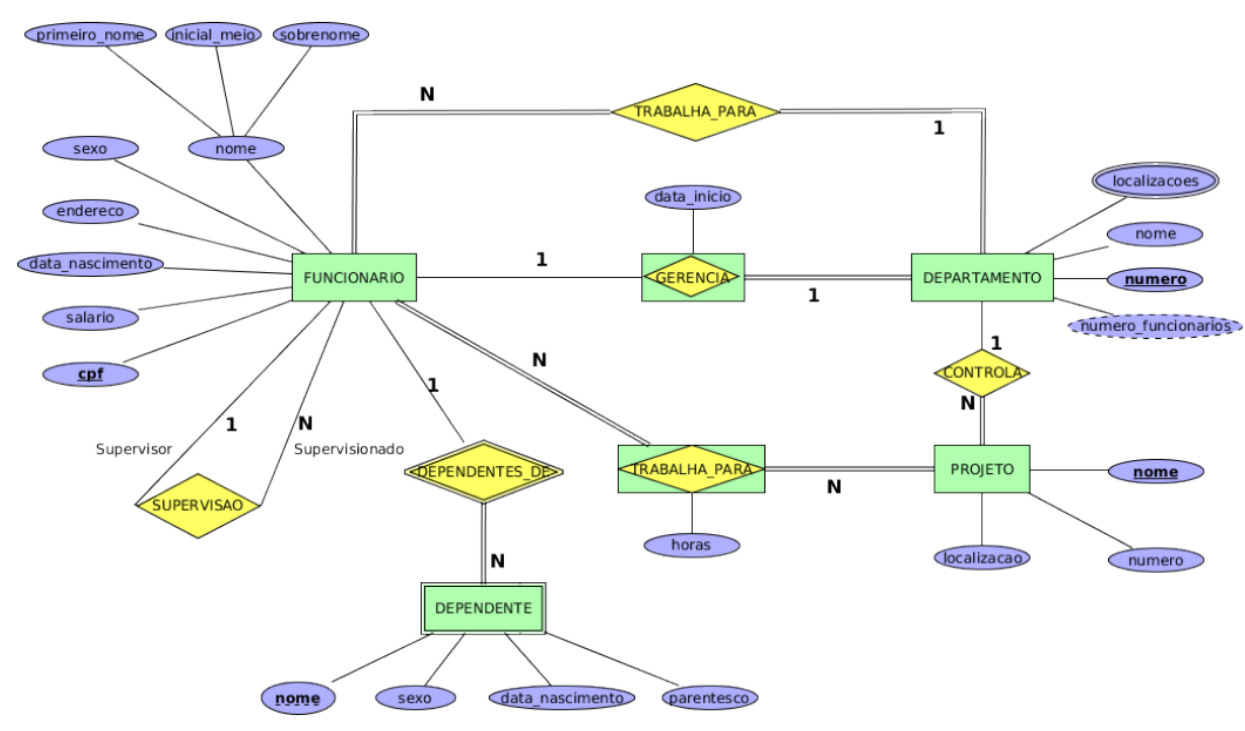

Figura 1. Diagrama ER do banco de dados de uma empresa

Atributos: Uma entidade é representada por um conjunto de atributos. Os atributos são propriedades descritivas que expressam características em comum de diferentes entidades em um conjunto de entidades. A designação de um atributo, para um conjunto de entidades, expressa que o banco de dados armazena informações semelhantes concernentes a cada entidade no conjunto de entidades; entretanto, cada entidade pode ter seu próprio valor para cada atributo [1]. Além disso, segundo Date [3], os atributos podem ser: (i) simples ou compostos; (ii) chaves; (iii) univalorados ou multivalorados; e (iv) básicos ou derivados. Em um modelo conceitual de alto nível, um atributo chave expressa uma restrição de unicidade, ou seja, cada entidade no conjunto de entidades contém valor distinto referente a esse atributo.

A Figura 1 ilustra cada um desses atributos. Por exemplo, a entidade FUNCIONARIO possui salario como atributo simples; nome como atributo composto, pois nome é composto de primeiro_nome, inicial_meio e sobrenome; e cpf como atributo chave. A entidade DEPARTAMENTO tem localizacoes como atributo multivalorado, pois um departamento pode-se encontrar em diferentes lugares, e numero_funcionarios como atributo derivado, ou seja, pode-se obter a quantidade de empregados através do somatório de todos os funcionários da entidade FUNCIONARIO.

Relacionamentos: Uma entidade pode se relacionar com outras entidades. Relacionamentos são conexões entre duas ou mais entidades [7]. As entidades envolvidas em determinado relacionamento são ditas participantes desse relacionamento. O número de participantes em determinado relacionamento é chamado grau desse relacionamento.

Seja $R$ um tipo de relacionamento que envolve o tipo de entidade $E$ como participante. Se toda instância de $E$ participa de pelo menos uma instância de $R$, então a participação de $E$ em $R$ é considerada total; do contrário ela é considerada parcial. Relacionamentos em diagramas ER podem ser modelados de diversas formas: um para um; um para muitos; ou muitos para muitos. Na Figura 1 , entidades do tipo DEPARTAMENTO 
podem se relacionar com entidades do tipo PROJETO da seguinte forma: um departamento controla pelo menos um projeto. Assim, esse exemplo expõe a existência de um relacionamento binário um para muitos $(1: \mathrm{N})$ com restrição de participação total no lado $\mathrm{N}$.

O Modelo ER proposto por Peter Chen em 1976 é uma forma simples de representar problemas do mundo real de forma bem próxima da descrição semântica dos dados e as relações entre eles. O Modelo ER é composto por elementos chamados de entidades, atributos e relacionamentos.

\subsection{Modelo ER Estendido}

Desde o final da década de 1970, projetistas de aplicações de banco de dados têm tentado projetar esquemas de banco de dados mais precisos, que reflitam as propriedades de dados e restrições com mais precisão [5]. Diante desse fato e do sucesso do paradigma orientado a objetos, em 1986, Teorey et al. [16] propuseram uma extensão ao modelo ER acrescentando os conceitos de relacionamentos de classe/subclasse, herança de tipo, especialização/generalização (também chamadas hierarquias) e suas restrições.

A Figura 2 exemplifica o conceito de generalização e especialização. A generalização se refere ao processo de definição de um tipo de entidade generalizado com base nos tipos de entidades dados. Nessa figura, pode-se também observar a especialização ao se modelar os tipos de entidades partindo da superclasse (tipo de entidade FUNCIONARIO) e seguindo para a especificação do conjunto de subclasses (SECRETARIA, TECNICO e ENGENHEIRO).

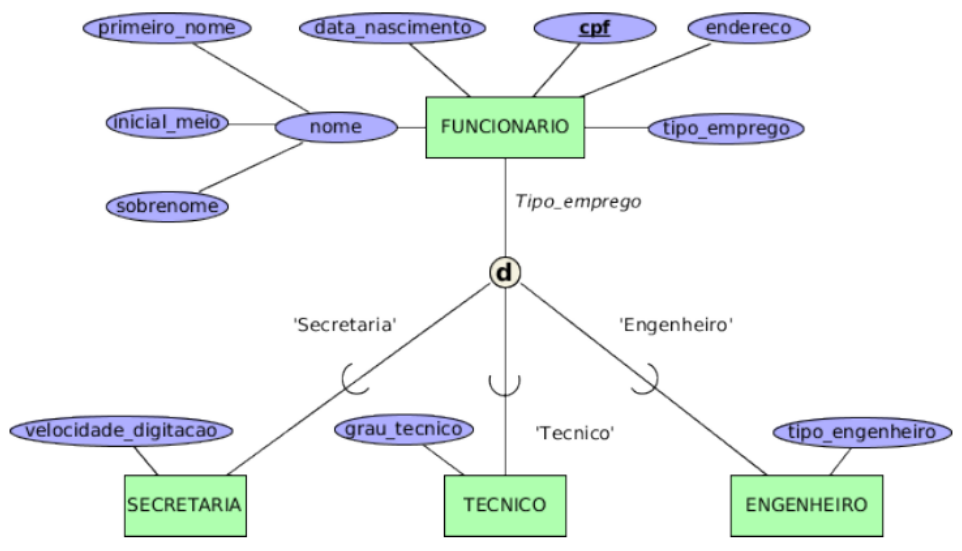

Figura 2. Diagrama EER de parte do banco de dados de uma empresa

Na Figura 2, por exemplo, uma entidade do tipo de entidade FUNCIONARIO pode ter seu emprego classificado como do tipo SECRETARIA, TECNICO ou ENGENHEIRO, o que caracteriza o tipo de entidade FUNCIONARIO como superclasse ou supertipo e, de forma análoga, os tipos de entidades SECRETARIA, TECNICO e ENGENHEIRO como subclasses ou subtipos. Vale ressaltar ainda que as entidades membros de uma subclasse (SECRETARIA, TECNICO e ENGENHEIRO) herdam todos os atributos do tipo de entidade da superclasse FUNCIONARIO).

Duas outras restrições podem se aplicar a uma especialização. A primeira é a restrição de disjunção, que especifica que as subclasses da especialização devem ser dis- 
juntas. Isso significa que cada entidade pode ser membro de, no máximo, uma das subclasses da especialização. Se nas subclasses não for imposta a restrição de disjunção, seus conjuntos de entidades podem ser sobrepostos, isto é, cada entidade pode ser membro de mais de uma subclasse da especialização. A Figura 2 apresenta um exemplo de especialização com restrição de disjunção, na qual o tipo do trabalho do FUNCIONARIO pode ser somente um dos tipos SECRETARIA, TECNICO, ou ENGENHEIRO.

A segunda restrição sobre a especialização é chamada de restrição de completude, que pode classificada como total ou parcial. Uma restrição de especialização total especifica que toda entidade da superclasse precisa ter como membro ao menos uma subclasse na especialização. Já a especialização parcial permite que uma entidade da superclasse não tenha como membro qualquer uma das subclasses [5]. Portanto, podemos classificar as restrições de especialização como quatro possíveis: (i) disjunção, total; (ii) disjunção, parcial; (iii) sobreposição, total; e (iv) sobreposição, parcial. A Figura 2 exemplifica o caso de especialização com restrição de disjunção e restrição de completude parcial (caso ii supracitado), onde o tipo do trabalho do FUNCIONARIO pode ser somente um dos tipos SECRETARIA, TECNICO, ou ENGENHEIRO, além de permitir não ter como membro nenhum dos três, já que a restrição de completude é parcial.

\subsection{TerraER}

O TerraER é uma ferramenta de código aberto, voltada ao meio acadêmico, mais especificamente no auxílio ao aprendizado de disciplinas de modelagem conceitual de banco de dados [12, 13]. TerraER permite criar modelos conceituais de alto nível mais condizentes ao que os professores lecionam na disciplina de banco de dados. Isso pode ser constatado através da Figura 3, na qual a barra de ferramentas de objetos possui atalhos para criação de elementos do diagrama ER na notação de Peter Chen e EER, adotada por Elmasri e Navathe [5].

Devido à facilidade na criação de diagramas que utilizam modelo conceitual de alto nível e pelo fato de a ferramenta TerraER já ser adotada em mais de 30 Instituições de Ensino Superior (IES), essa foi escolhida para implementar o módulo de validação de Modelos ER, de forma a auxiliar professores e alunos no projeto e correção de atividades e tornar o processo de aprendizado mais rápido e interativo para o aluno.

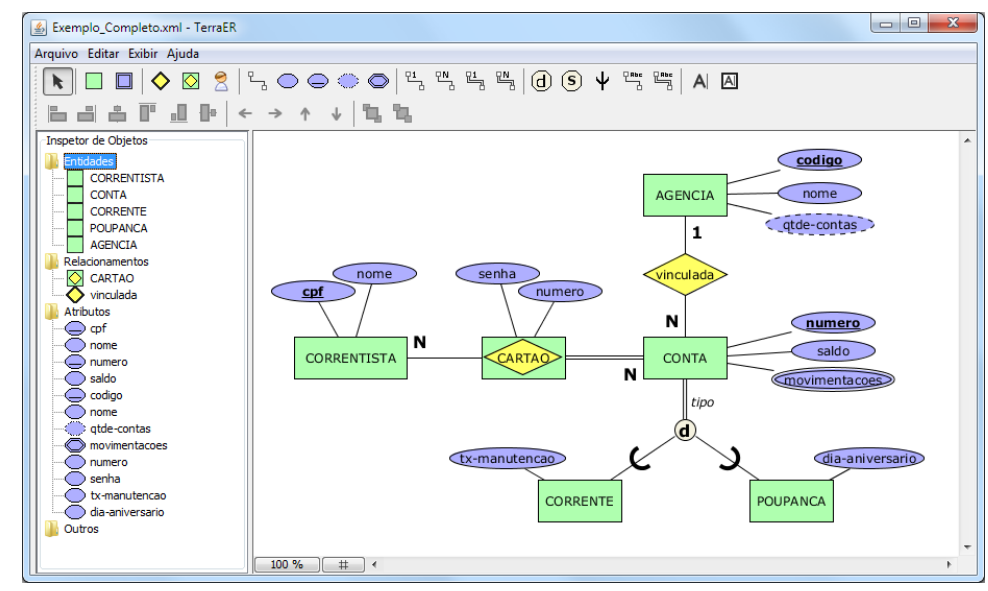

Figura 3. Modelo ER estendido na ferramenta TerraER 


\section{Validação de Modelos ER}

Esta seção descreve a solução proposta para validação de Modelos ER. As Seções 3.1 e 3.2 formalizam cada elemento ER e as suas construções válidas, respectivamente. A Seção 3.3 descreve a integração da validação proposta na ferramenta TerraER. Por fim, a Seção 3.4 avalia a solução proposta sob a perspectiva educacional.

\subsection{Elementos}

Os elementos se dividem em duas categorias: figuras e conexões. Por um lado, as figuras são partes do modelo que existem por si só, como entidades, atributos, relacionamentos e o tipo de herança. Por outro lado, as conexões são elementos que necessitam de pelo menos duas figuras para existir, como conexões de atributos, conexões de participação e cardinalidade dos relacionamentos e conexões relacionadas à herança. A Tabela 1 ilustra os elementos ER usados na validação e suas categorias.

Tabela 1. Elementos ER

\begin{tabular}{|c|c|c|c|c|c|c|}
\hline \multicolumn{3}{|c|}{ Figuras } & \multicolumn{4}{|c|}{ Conexões } \\
\hline & Entidade & Entidade Fraca & 담 & Conexão de Atributo & 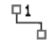 & Opcional '1 para' \\
\hline$\theta$ & Entidade Relacionamento & Relacionamento & 占 & Obrigatória '1 para' & 吅 & Opcional 'n para' \\
\hline$\otimes$ & Relacionamento Fraco & Atributo & 足 & Obrigatória 'n para' & 믈 & Geral opcional \\
\hline$\Leftrightarrow$ & Atributo Chave & Atributo Chave Parcial & 驾的 & Geral obrigatória 'n para' & $\psi$ & Generalização \\
\hline & Atributo Derivado & Atributo Multivalorado & & & & \\
\hline d & Disjunção & Sobreposição & & & & \\
\hline u & União & & & & & \\
\hline
\end{tabular}

\subsection{Formalização das Conexões Válidas}

Claramente, alunos não podem realizar qualquer tipo de conexão entre quaisquer duas figuras. A Figura 4 ilustra um grafo onde os vértices são figuras ou grupo de figuras e as arestas são rotuladas com as conexões válidas entre elas.

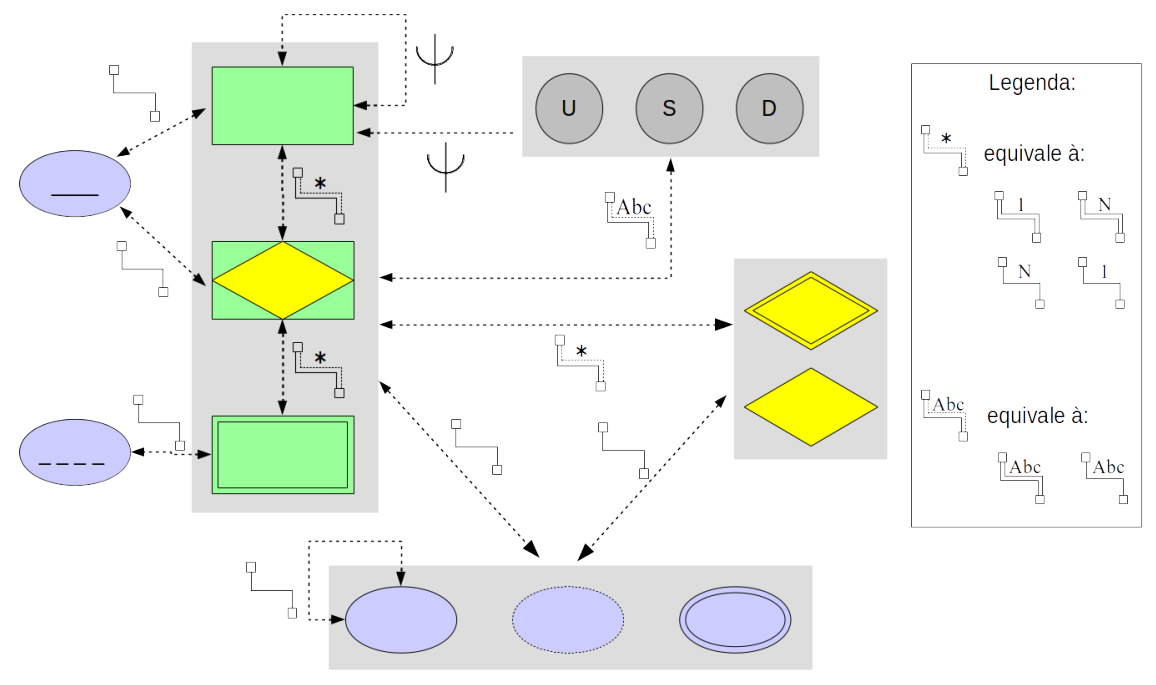

Figura 4. Conexões válidas

Conforme supramencionado, figuras foram devidamente agrupadas para facilitar a visualização. Um grupo de figuras é representado por um retângulo cinza em volta 
das figuras e significa que todas elas compartilham as mesmas conexões válidas. Por exemplo, a conexão de atributo pode ser estabelecida entre (i) qualquer elemento do grupo composto por entidade, entidade fraca e entidade relacionamento e (ii) qualquer elemento do grupo composto por atributo, atributo derivado e atributo multivalorado.

No entanto, um elemento pertencente a um grupo pode ter conexões válidas que não são compartilhadas com os demais elementos do grupo. Por exemplo, a entidade e entidade relacionamento permitem conexão com atributo chave, porém entidade fraca mesmo pertencendo ao mesmo grupo de figuras - não permite.

De forma similar, conexões também foram agrupadas de forma a facilitar a visualização em quatro grupos principais:

- Conexão de atributo (grupo unitário);

- Conexão de relação (grupo composto pela conexão opcional e obrigatória '1 para', e conexão opcional e obrigatória ' $N$ para');

- Conexão geral (grupo composto pela conexão geral opcional e obrigatória); e

- Conexão de generalização (grupo unitário).

Por exemplo, o grupo composto por entidade, entidade fraca e entidade relacionamento pode estabelecer qualquer conexão de relação (conexão opcional ou obrigatória '1 para', ou conexão opcional ou obrigatória ' $\mathrm{N}$ para') com o grupo composto por relacionamento e relacionamento fraco.

\subsection{Projeto e Implementação}

Durante a modelagem na ferramenta TerraER, o aluno seleciona as figuras (ver lado esquerdo da Tabela 1) desejadas e as posiciona no modelo. Consequentemente, o aluno as conecta usando qualquer conexões entre figuras (ver lado direito da Tabela 1). O módulo de validação proposto atua exatamente no momento em que o aluno conecta duas figuras. Basicamente, o método validateLineConnection da classe ModelValidation ${ }^{6}$ é responsável por verificar se os elementos podem ser conectados.

Caso a conexão não seja permitida entre tais figuras, TerraER destacará a conexão na cor vermelha no intuito de alertar o aluno sobre o erro encontrado. Mais importante, o aluno não só é notificado do problema como pode solicitar sugestão de como corrigi-lo. Uma tela de recomendações, como a ilustrada na Figura 5, aponta quais as possíveis conexões entre as tais duas figuras e também quais as possíveis figuras que podem ser conectadas usando tal conexão.

Primeiramente, é exibida uma lista de conexões válidas entre os dois elementos envolvidos no erro encontrado. Por exemplo, conforme ilustrado na Figura 5, uma entidade está sendo erroneamente conectada à uma outra entidade por meio de uma conexão de relação. Nesse caso, é exibido ao aluno que a única conexão válida entre duas entidades é a conexão de generalização.

\footnotetext{
${ }^{6}$ Essa classe está publicamente disponível em: https://github.com/rterrabh/terraer/blob/master/terraer_ project/src/main/java/org/jhotdraw/draw/ModelValidation.java
} 


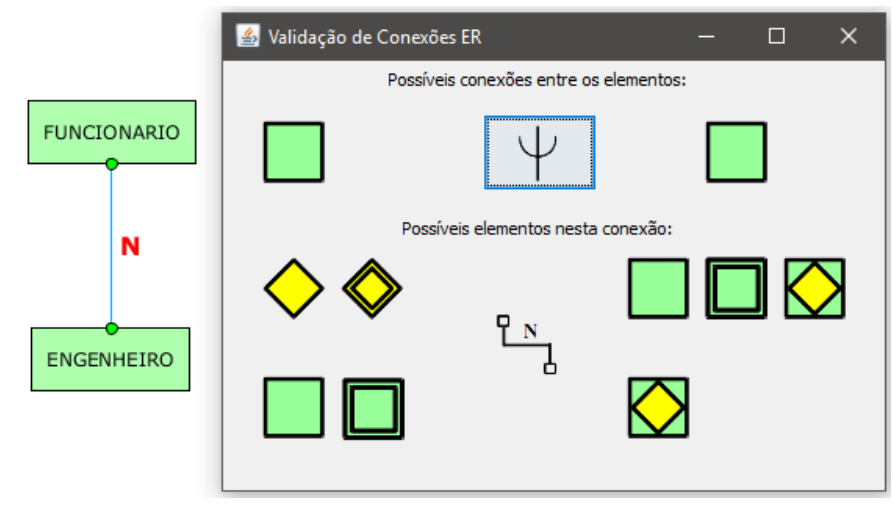

Figura 5. Conexão inválida em vermelho e tela de recomendações no TerraER

Segundamente, é exibida uma lista de elementos que podem ser conectados usando a conexão em que há o erro (conexão de relação, nesse exemplo). Por exemplo, conforme ilustrado na Figura 5, a conexão de relação pode ocorrer (i) relacionamento ou relacionamento fraco com entidade, entidade fraca ou entidade relacionamento, e (ii) entidade ou entidade forte com entidade relacionamento.

Como trabalho futuro, pretende-se integrar às recomendações explicações de uso de cada um dos elementos ER. O objetivo é trazer informações que podem - de forma complementar - contribuir para o aprendizado do aluno.

\subsection{Avaliação sob a Perspectiva Educacional}

Esta seção avalia a solução proposta sob a perspectiva educacional. Três elementos positivos puderam ser observados: (i) a liberdade do aluno de errar e notificá-lo sobre o erro, (ii) a facilidade de correção pelo professor e (iii) a influência da estratégia de tentativa-e-erro.

Permitir o aluno errar e fornecer feedback: Sabe-se que sinapses são geradas ao se cometer erros [10]. Mesmo que não se tenha ciência do erro cometido, sinais ERN (Negatividade Relacionada ao Erro) são gerados [9]. Porém, quando se tem ciência do erro por meio de algum feedback, sinais ERP (Positividade Relacionada ao Erro) são gerados pouco depois (200 a 500 milissegundos) [6, 11].

Estudos afirmam que a presença desse sinal de ERP está relacionada ao aprendizado pós-erro, levando a um melhor desempenho e precisão em atividades futuras [14, 8]. De acordo com a teoria supracitada, a presença do ERP está relacionada tanto ao cometimento de erro quanto ao feedback pós-erro. Logo, é seguro afirmar que permitir o erro do aluno e notificá-lo potencialmente melhora o seu aprendizado na tarefa realizada.

O fato de o feedback proporcionar maior presença do ERP [6, 11] explica ser amplamente utilizado na educação e no processo de aprendizado, especialmente o feedback corretivo [4, 15]. Tipicamente envolve o estudante recebendo um feedback formal ou informal sobre a execução de uma ou mais tarefas. A solução proposta envolve, no momento de cada nova conexão construção do modelo, uma verificação dessa construção. Caso houver algum erro de construção, o aluno é notificado através de um feedback corretivo. Essa notificação pode ser considerada um feedback imediato, isso por trazer à ciência do aluno o erro no momento em que este é cometido, o que pode 
aprimorar ainda mais o aprendizado [4]. Ao permitir o erro do aluno e, imediatamente em seguida, informá-lo do mesmo, é instigado o aprendizado a fim de evitar tal erro em atividades futuras.

Menor preocupação com detalhes do modelo: Um dos objetivos do módulo de validação proposto é otimizar o processo educacional. Notificar e corrigir esses erros de construção facilitam a construção do modelo como um todo, uma vez que permite que o aluno foque na tarefa de modelagem conceitual, sem preocupações com formalizações do modelo. Isso ocorre sem sacrificar o aprendizado das formalizações do modelo, uma vez que o sistema de feedback provê exatamente isso. Ainda, essa facilitação também é transferida ao professor que poderá corrigir atividades focado exclusivamente na modelagem conceitual. Enfim, o fato de não ter que verificar o modelo fomenta uma maior eficiência no processo de educação.

Tentativa-e-erro: O módulo de validação pode acabar induzindo alunos a abusar da tentativa-e-erro. Sob a perspectiva educacional, não é interessante que o aluno tente diversas conexões até que alguma seja válida. O módulo de validação proposto ajuda a minimizar esse problema, uma vez que permite ao aluno (mas o alerta de) estabelecer conexões inválidas. Ao alertar que são inválidas, o módulo de validação apresenta as conexões válidas. A observação da lista de conexões válidas logo após o erro instiga o aluno a refletir sobre a correta de acordo com a modelagem do problema. Mas é importante ressaltar que o problema - mesmo minimizado - pode ainda ocorrer já que o aluno pode simplesmente estabelecer conexões inválidas e aceitar as sugestões sem prévia análise.

\section{Considerações Finais}

Devido à sua simplicidade em representar dados e relações, o modelo EntidadeRelacionamento (ER) é largamente adotado no ensino de modelagem de dados conceitual. No entanto, durante o processo de aprendizagem, muitos dos erros cometidos pelos alunos não estão relacionados à lógica do modelo, mas sim, às suas construções. Este artigo reivindica que alunos devem focar na tarefa de modelagem conceitual, sem preocupações com formalizações do modelo.

Diante disso, este artigo propôs uma validação de modelos ER integrada à uma ferramenta acadêmica de modelagem, denominada TerraER. Esse módulo de validação é capaz de verificar conexões logo que são adicionadas ao modelo e - caso inválidas - não só notificar o aluno como sugerir as conexões válidas por meio de uma tela de recomendação que aponta quais as possíveis conexões entre os elementos e ainda quais os possíveis elementos que podem ter tal conexão estabelecida.

Como contribuição, a o módulo de validação proposto neste artigo visa tornar o processo de aprendizado mais rápido e interativo para o aluno, além de reduzir o esforço de correção pelo professor. Isso se justifica, primeiramente, pelo fato de o módulo de validação permitir o erro do aluno e imediatamente o notificar; cenário o qual instiga o aprendizado e evita a recorrência do mesmo erro. Em segundo lugar, o aluno pode se preocupar exclusivamente na tarefa de modelagem conceitual, sem preocupações com formalizações do modelo. Da mesma forma, professores, no processo de correção, podem ter a atenção voltada exclusivamente à modelagem, o que fomenta maior eficiência do processo educacional. 
Como trabalho futuro, pretende-se auditar o módulo de validação para que o professor possa ter acesso aos erros cometidos pelos alunos e a frequência dos mesmos. Isso viabilizará a compreensão das dificuldades reais do aluno e consequentemente trará benefícios ao processo educacional. Pretende-se também avaliar o módulo de validação proposto em relação à sua efetividade e conduzir uma comparação com outros trabalhos.

Agradecimentos: Este trabalho foi apoiado pela FAPEMIG, CAPES e CNPq.

\section{Referências}

[1] S. A. Korth H. F. and S. Sudarshan. Sistema de Banco de Dados. Elsevier, 5th edition, 2006.

[2] P. P.-S. Chen. The entity-relationship model - toward a unified view of data. ACM Transactions on Database Systems, 1(1):9-36, 1976.

[3] C. J. Date. Introdução a Sistemas de Banco de Dados. Elsevier, 8th edition, 2004.

[4] R. Ellis. Corrective feedback and teacher development. L2 Journal, 1(1):3-18, 2009.

[5] R. Elmasri and S. B. Navathe. Sistemas de Banco de Dados. Pearson Addison Wesley, 6th edition, 2011.

[6] M. Falkenstein, J. Hoormann, S. Christ, and J. Hohnsbein. ERP components on reaction errors and their functional significance: a tutorial. Biological Psychology, 51(2):87$107,2000$.

[7] H. Garcia-Molina. Database Systems: The Complete Book. Pearson Prentice Hall, 2nd edition, 2009.

[8] R. Hester, N. Barre, J. B. Mattingley, J. J. Foxe, and H. Garavan. Avoiding another mistake: error and posterror neural activity associated with adaptive posterror behavior change. Cognitive, Affective, \& Behavioral Neuroscience, 7(4):317-326, 2007.

[9] S. J. Luck. An introduction to the event-related potential technique. cognitive neuroscience. The Quarterly Review of Biology, 81(2):201-202, 2006.

[10] J. Moser, H. Schroder, C. Heeter, T. Moran, and Y.-H. Lee. Mind your errors. Psychological Science, 22(12):1484-9, 2011.

[11] S. Nieuwenhuis, K. R. Ridderinkhof, J. Blom, G. P. Band, and A. Kok. Error-related brain potentials are differentially related to awareness of response errors: Evidence from an antisaccade task. Psychophysiology, 38(5):752-760, 2001.

[12] H. Rocha and R. Terra. Uma ferramenta voltada ao ensino do modelo de entidaderelacionamento. VI Escola Regional de Banco de Dados (ERBD), pages 1-4, 2010.

[13] H. Rocha and R. Terra. TerraER - an academic tool for er modeling. Methods and Tools, 1(3):38-41, 2013.

[14] H. S. Schroder, M. E. Fisher, Y. Lin, S. L. Lo, J. H. Danovitch, and J. S. Moser. Neural evidence for enhanced attention to mistakes among school-aged children with a growth mindset. Developmental Cognitive Neuroscience, 24(1):42-50, 2017.

[15] G. Steuer, G. Rosentritt-Brunn, and M. Dresel. Dealing with errors in mathematics classrooms: Structure and relevance of perceived error climate. Contemporary Educational Psychology, 38(3):196-210, 2013.

[16] T. J. Teorey, D. Yang, and J. P. Fry. A logical design methodology for relational databases using the extended entity-relationship model. ACM Computing Surveys, 18(2):197222, 1986. 